Research report

\title{
Changes in fine motor retardation in depressed patients treated with fluoxetine
}

\author{
Bernard Sabbe $^{\mathrm{a}, *}$, Jacques van Hoof ${ }^{\mathrm{b}}$, Wouter Hulstijn ${ }^{\mathrm{c}}$, Frans Zitman ${ }^{\mathrm{d}}$ \\ "Department of Psychiatry, University of Nijmegen, P.O. Box 9101.6500 HB Nijmegen, The Netherlands \\ ${ }^{\mathrm{b}}$ Institute of Psychiatric Care, Rosmalen, Rosmalen, The Netherlands \\ ${ }^{c}$ Nijmegen Institute for Cognition and Information (NICI), Unicersity of Nijmegen, Nijmegen. The Netherlands \\ ${ }^{\mathrm{d}}$ Department of Psychiatry, Unitersity of Nijmegen. Nijmegen. The Netherlands
}

Received 16 January 1996; revised 20 May 1996; accepted 20 May 1996

\begin{abstract}
Changes in psychomotor slowing were studied in 21 inpatients with a Major Depressive Episode. Fine motor retardation was measured and analysed using computer-aided drawing and figure-copying tasks at T0 (the start of 6 weeks treatment with fluoxetine $20 \mathrm{mg} /$ day) and 5 weeks later (T1). The differences in reaction time between the patients and a group of healthy, matched controls at T0 had disappeared at T1. The initial motor deficit, expressed in longer movement times, had not improved at $\mathrm{T} 1$. These findings combined with the effect of manipulation of cognitive and motor demands, suggested that only cognitive processes had accelerated.
\end{abstract}

Keywords: Psychomotor retardation; Depression; Figure drawing; Movement analysis: Antidepressant treatment; Fluoxetine

\section{Introduction}

Throughout the past decades psychomotor retardation has consistently been reported as a predictor of antidepressant response (Joyce and Paykel, 1989). However, relevant research data are scarce, mainly because of the lack of reliable methods to quantify psychomotor retardation. In follow-up studies that used a clinical rating scale, such as the Salpêtrière Retardation Rating Scale, correlations were found between the severity of the retardation before treatment and the differences in the scores on the Hamil-

\footnotetext{
* Corresponding author.
}

ton Depression Rating Scale at the start of treatment and 4 weeks later (Widlöcher, 1983a). It was noticed in actometrical studies that the activity level progressively increased during treatment, while the duration of immobility decreased during clinical improvement (Royant-Parola et al., 1986). In studies that used choice reaction time tasks, both a motor and a cognitive component seemed to contribute to psychomotor slowing in the depressed patient. The decision time or the matching time decreased significantly during clinical improvement, which suggested cognitive improvement, while 'pure' motor retardation remained unchanged (Ghozlan and Widlöcher, 1989; Rogers et al., 1987).

New technologies allow the more specific and detailed analysis of aspects of retardation and the 
way they change during treatment. The first reports stem from speech research. Earlier studies (Greden and Carroll, 1980; Greden et al., 1981; Hoffmann et al., 1985) had reported that antidepressant chemotherapy shortened the speech pause time during clinical improvement. Recently, Kuny and Stassen (1993), using advanced techniques, found that several prominent features of speaking behaviour and voice characteristics were closely related to the time course of recovery from depression.

In our previous studies we measured and analysed fine motor retardation in depressed patients at the start of treatment and compared the results to those of normal control persons (Van Hoof et al., 1993; Van Mier and Hulstijn, 1993; Sabbe et al., 1996). It was concluded that compared to the normal control subjects, the writing and drawing behaviours of the large majority of depressed patients were slowed, although great inter-individual differences were present. This manifested itself when they drew lines and simple and complex figures. Both the reaction time and movement time were prolonged, which suggested that fine motor retardation was the result of slowing of cognitive and motor processes.

In this study, following the preliminary research of Van Hoof et al. (1993), we examined the changes in depressive retardation at the start of treatment with antidepressive medication and at the end of the course 5 weeks later, in order to answer the following questions:

1. Does fine motor slowing in the patient group diminish or disappear after treatment?

2. If slowing improves after treatment, is it the cognitive and/or the motor slowing that improves?

\section{Method}

\subsection{Subjects}

Forty-two subjects participated in the study: 21 patients with a Major Depressive Episode (MDE) and 21 normal control subjects. In the study period all the MDE patients had been hospitalized at the Clinic of Psychiatry of the University Hospital Nijmegen, the Netherlands. All the patients aged between 18 and 65 years with an MDE and a minimum score of 18 on the Hamilton Depression Rating Scale (Hamilton, 1960), who were admitted between September 1992 and April 1994, were asked for informed consent after the nature of the study had been fully explained to them. Patients were excluded if they met one of the following criteria: motor disabilities affecting writing behaviour, severe cardiovascular or hepatic disease, renal failure and previous unsuccessful treatment with fluoxetine. The group comprised 11 male and 10 female patients. All the patients had a DSM-III-R (American Psychiatric Association, 1987) diagnosis of a Major Depressive Episode, single episode (296.2) or recurrent (296.3); only 1 patient had a Bipolar Disorder, Depressed (296.5). The episode was severe in all the patients. Six patients did have psychotic features (code 4) and of the 15 patients that remained, 11 met the criteria of major depression, melancholic type. Two patients had a subsidiary diagnosis of previous alcohol and benzodiazepine dependence. Three patients displayed a clinical state of agitation. For each patient there was a control subject, matched for age, sex and educational level.

\subsection{Procedure and tasks}

Once admitted to the study, all antidepressant drugs were stopped and any other psychotropic drugs were reduced as much as the condition of the patient allowed. Then fluoxetine $20 \mathrm{mg}$ /day was administered to the MDE patients for 6 weeks. The tests were performed 1 week later (T0) and after 6 weeks (T1) of treatment. During this period, changes in the medication regimen were kept to a strict minimum. In fact, only very low doses of anxiolytics or neuroleptics, used by the patients with psychotic features $(n=6)$, were allowed. For a discussion of the possible positive or negative effects of the use of this medication on the tasks used, see Sabbe et al., 1996.

Three clinical rating scales were scored at $\mathrm{T} 0$ and T1: the Hamilton Depression Rating Scale (Hamilton, 1960), the Zung Self-Rating Scale (Zung, 1965) and the Salpêtrière Retardation Rating Scale (Widlöcher, 1983b). Tests consisted of a series of copying tasks with the aid of a pen on a graphics tablet. The movement registration method is discussed in the next paragraph. Stimuli differed in complexity and for task II also in familiarity (Fig, 1). 
TASK I

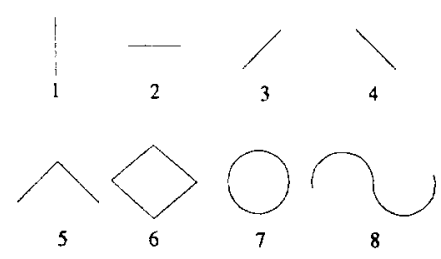

TASK II

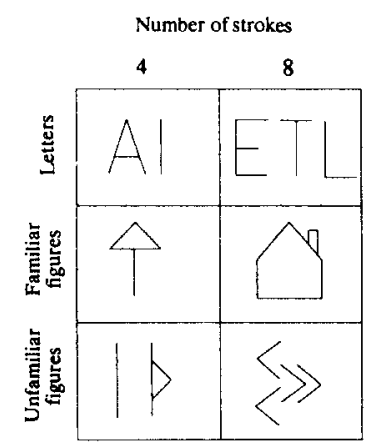

TASK III

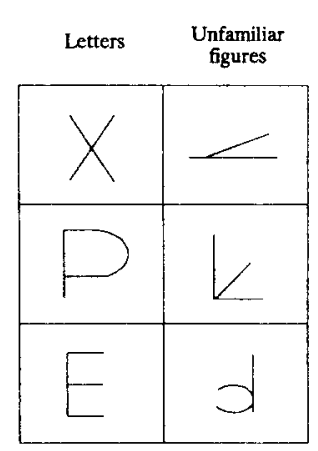

Fig. 1. Stimulus designs used in Tasks I, II and III.

Complexity was defined as the number of strokes in a figure; familiarity could be perceptual or motor. For example, letters are well-known perceptually and motorically, i.e., subjects are used to writing them, while figures, such as an arrow or a house, are well-known perceptually but not motorically; novel and nonsense patterns are unknown perceptually and motorically.

Three copying tasks were used:

- Task I analysed the degree of retardation in the drawing of lines and simple figures. The patients and controls had to copy 4 straight lines (one vertical, one horizontal and two diagonals) and 4 simple figures (Fig. 1). All the stimuli were presented six times in random order. The lines could be divided on a cognitive level, i.e., in visuo-spatial processing and planning, in two classes: the vertical and horizontal lines were easier to draw than the two diagonal lines. In the simple figures the angle and circle were less complex than the diamond and spiral.

- The degree of complexity of the figures was increased in task II. Three types of stimulus had to be copied: combinations of capital letters, fa- miliar figures and novel, nonsense patterns (Fig. 1). Four stimuli of each type were presented with differing complexity, two with 4 strokes and two with 8 strokes.

- In task III a specific type of manipulation was executed that focused on visuo-spatial processing. Eight figures, 4 combinations of letters and 4 figures (Fig. 1) were presented, with the instruction to copy them at another angle, i.c. having rotated them through 90 degrees to the right.

Different sets of figures were presented at T0 and T1 to avoid a learning effect in tasks II and III. Afterwards it appeared that although they were assigned at random, the figures presented in task II at T1 were more difficult than those presented in the corresponding task at $\mathrm{T} 0$.

In all the tasks, the participants received the standard instruction, as usual in this type of research, to draw as quickly and as accurately as possible. As soon as the pen touched the paper, the stimulus disappeared from the video viewing screen. In tasks II and III the subject could reinspect the figure by touching the pen against a red spot at the lower right hand corner of the digitiser. He/She was asked to do this only as an 'emergency' measure, if he/she felt that otherwise too many errors would be made.

Prior to each task, a practice session was given in which the participants could become accustomed to the writing tablet and the procedure.

The investigators worked independently of the treatment staff. The three tasks were performed in a fixed order in 30 to $60 \mathrm{~min}$. They all took place between 2 and 5 p.m., to avoid possible influences of circadian rhythm.

\subsection{Recording and analysis}

The drawing movements were recorded using a Calcomp 2300 digitiser, connected to a PC (63S386) that had been specially designed to measure pen pressure, with a precision of $2 \mathrm{~g}$ (Maarse et al., 1988).

The position of the pen on the graphics tablet and the axial pen force were recorded with a frequency of $100 \mathrm{~Hz}$ and a resolving power of $0.2 \mathrm{~mm}$ and $1 \mathrm{~g}$. respectively. The following movement variables were measured: total time (TT), i.e., the sum of the reaction time and the movement time; reaction time 
(RT), i.e., the time interval between the presentation of the stimulus and the moment the pen touched the paper and the pressure threshold was exceeded; movement time (MT), i.e., the time interval between the first moment and last moment that the pressure threshold was exceeded. Movement time (MT) was divided into the time that the pen was on the paper and the pressure threshold was exceeded (movement time pen down: MTdown), the time that the pen was above the paper and the pressure was below the threshold (movement time pen up: MTup) and the video reinspection time (MTvideo), i.e., the length of time that the figure was reinspected.

The drawings in task II and task III were scored for errors by two independent investigators. Classification was made as follows:

A-type error. The drawing more or less resembled the actual stimulus. Distinction was made between small and severe errors. A1: rotation error of up to 30 degrees, distortion in proportions or in relations, segmentation of parts of the stimulus, fragmentation and alignment, omissions and additions (all up to $1 / 3$ of the number of strokes of the original stimulus); also the drawings in which corrections had been made. A2: rotation of more than 30 degrees; two-dimensionality instead of three-dimensionality; wrong letters(s); omissions, additions, reversal and distortion in form (all up to $2 / 3$ of the number of strokes of the original stimulus).

$B$-type error. The original stimulus could not be recognized because of too many omissions or additions, or no copy had been made.

Statistical analysis was performed with analyses of variance for each variable according to a repeated measurement design. The within-subject factors were complexity (task I), complexity and familiarity (task II) and familiarity (task III), while the between-subject factor was group (patients vs. controls).

\section{Results}

\subsection{Clinical rating scales}

The mean scores of the patient group on the three clinical rating scales at $\mathrm{TO}$ and $\mathrm{T} 1$ were 24.7 (SD:
5.9) and 16.9 (SD: 9.2) $(P=0.000)$ for the Hamilton Depression Rating Scale, 62.9 (SD: 6.7) and 50.9 (SD: 14.8) $(P=0.001)$ for the Zung Self-Rating Scale and 26.0 (SD: 5.6) and 18.6 (SD: 11.9) $(P=$ $0.01)$ for the Salpêtrière Retardation Rating Scale. On this latter scale the means of the subscores for the motor items, the speech items and the cognitive items at $\mathrm{T} 0$ where $1.4,1.3$ and 1.8 , respectively, while at $\mathrm{T} 1$ they were $1.0,0.9$ and 1.3 , respectively.

\subsection{Copying tasks}

The results are presented task by task. Within each task, both the questions are discussed separately. To answer the first question about whether the retardation detected in the patient group diminished or even disappeared after treatment, the differences in the kinematic variables between the patient group and the control group (group effect) and the differences between $\mathrm{T} 0$ and $\mathrm{T} 1$ (session effect) are presented. Obviously, the interactions between the group effects and the session effects contain the central information to answer this question.

The second question about which component (cognitive or motor) improved if there was a positive treatment response, was investigated in two ways: by manipulating the complexity and familiarity within and between the subsequent tasks, and by making a detailed analysis of the different kinematic variables.

\subsubsection{Task I: Lines and simple figures}

The mean reaction and movement times at $\mathrm{T} 0$ and $\mathrm{T} 1$ are presented in Fig. 2, the outcome of the analyses of variance in Table 1 .

Lines. The reaction times in the patient group and the control group differed significantly and there was also a significant session effect. Fig. 2 shows a reduction of the reaction time of the patient group after treatment, while no changes occurred in the control group. The group by session interaction was significant. It also appeared that the patients' decrease in reaction time between $\mathrm{T} 0$ and $\mathrm{T} 1$ was greater for the diagonal lines than for the vertical and horizontal lines (line type by session interaction within patient group: $F=6.65, P=0.01$ ). The mean movement time in the patient group at $\mathrm{T} 0$ was one

Fig. 2. Mean reaction times (RT) and mean movement times (MT) of the depressed patients and the controls at T0 and T1 for copying lines and simple figures in Task I, complex figures in Task II and rotated figures in Task III. 
Task I: Lines
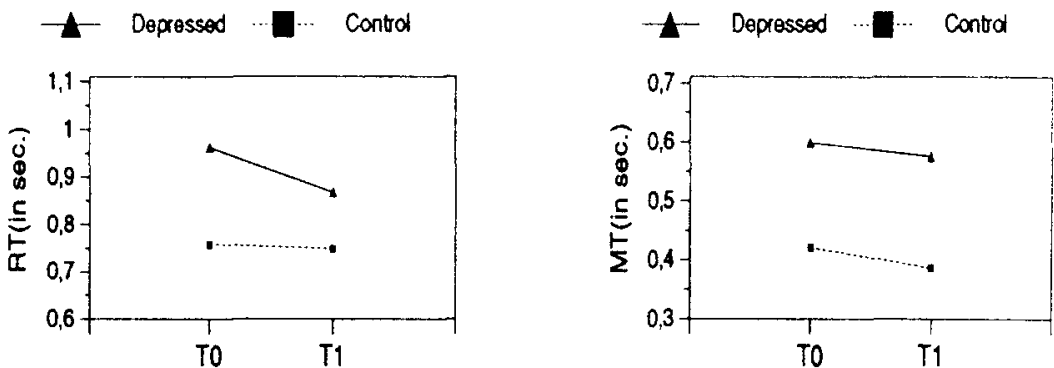

Task I: Simple Figures
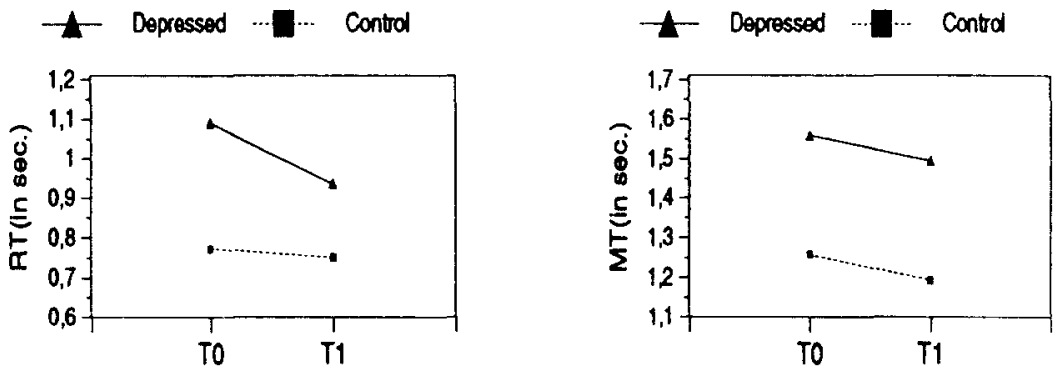

Task II: Complex Figures
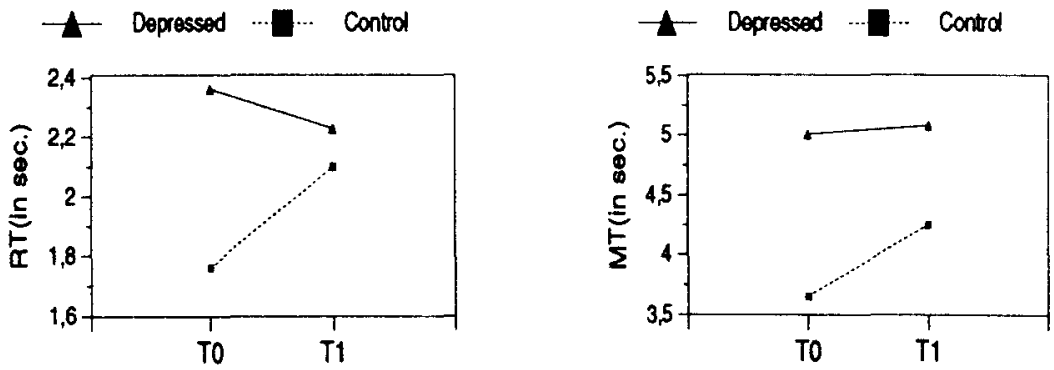

Task III: Rotation
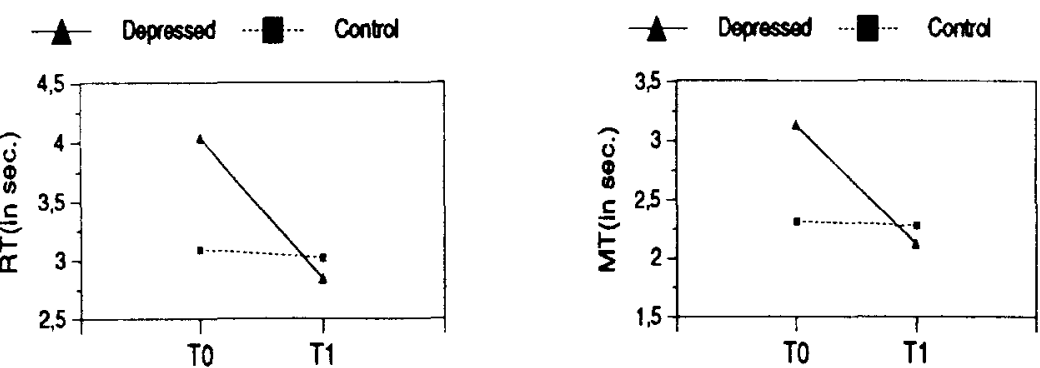
and a half times longer than that in the control group, but did not decrease significantly after treatment.

Simple figures. The results for copying simple figures were similar to those for drawing lines (Fig. 2 ). The group differences in reaction time were significant. The reduction in reaction time in the patient group after treatment did not occur in the control group. This was reflected in the significant group by session interaction. Additonal analyses revealed that the decrease in reaction time was more apparent in the more complex figures (diamondspiral) than in the simpler figures (angle-circle); the interactions between group and complexity ( $F=$ 5.87, $P=0.020$ ), and between group, complexity and session $(F=6.92, P=0.012)$ were significant. There was a significant group difference in movement time, but no significant decrease over session.

\subsubsection{Task II: Complex figures}

The mean reaction and movement times at $\mathrm{T} 0$ and $\mathrm{T} 1$ are presented in Fig. 2. In Fig. 3 the effect of increasing complexity is shown: the mean reaction times at $\mathrm{T} 0$ and $\mathrm{T} 1$ for the letters, figures and patterns are presented separately. The changes in the different kinematic variables from $\mathrm{T} 0$ to $\mathrm{T} 1$ are shown in Fig. 4. The outcome of the statistical tests can be found in Table 1 .

The reaction time in the control group at $\mathrm{Tl}$ was longer than at $\mathrm{T} 0$. In contrast with the simple figures that were the same at $\mathrm{T} 1$ and $\mathrm{T} 0$, the complex figures were dissimilar at $\mathrm{T} 1$ and $\mathrm{T} 0$. Post hoc, the figure set presented at $\mathrm{T} 1$ appeared to be more difficult than that presented at T0. Despite this, the reaction time in the patient group decreased slightly between $\mathrm{T} 0$ and $\mathrm{T} 1$. The significant interaction between group and session showed that the difference between patients and controls at T0 was significantly reduced at $\mathrm{T} 1$. The relative decrease in the reaction time in the patient group was more apparent for the more complex figures ( 8 lines versus 4 lines; Fig. 3). There was a significant interaction between group, complexity and session. Familiarity did not seem to be a relevant factor in this respect.

The movement time in the patient group was

Table 1

Results of the analyses of variance of tasks, I, II and III

\begin{tabular}{|c|c|c|c|c|c|c|}
\hline & $\mathrm{df}$ & RT & MT & MTup & MTdown & MTvideo \\
\hline \multicolumn{7}{|l|}{ Task I (lines) } \\
\hline Group (G) & 1 & $6.43^{*}$ & $10.24^{* *}$ & & & \\
\hline Session (S) & 1 & $4.94^{*}$ & 1.04 & & & \\
\hline $\mathrm{G} \times \mathrm{S}$ & 1 & $3.37^{\#}$ & 0.01 & & & \\
\hline \multicolumn{7}{|c|}{ Task I (simple figures) } \\
\hline Group $(\mathrm{G})$ & 1 & $7.09^{*}$ & $4.55^{*}$ & & & \\
\hline Session (S) & 1 & $7.09^{*}$ & $205.36^{* * *}$ & & & \\
\hline $\mathrm{G} \times \mathrm{S}$ & 1 & $4.31 *$ & 0.01 & & & \\
\hline \multicolumn{7}{|c|}{ Task II (complex figures) } \\
\hline Group (G) & 1 & 2.11 & $4.52^{*}$ & 0.13 & $3.699^{\#}$ & $5.93 *$ \\
\hline Complexity (C) & 1 & $87.32^{* * *}$ & $282.82^{* * *}$ & $246.23^{* * *}$ & $234.54 * * *$ & $33.02^{* * *}$ \\
\hline Session (S) & 1 & 1.79 & $4.81^{*}$ & $4.74^{*}$ & 1.06 & 2.48 \\
\hline $\mathrm{G} \times \mathrm{C}$ & 1 & 0.06 & $6.25^{*}$ & 0.45 & $3.51^{\#}$ & $7.73 * *$ \\
\hline $\mathrm{G} \times \mathrm{S}$ & 1 & $4.15^{*}$ & 1.32 & $8.66^{* *}$ & 0.21 & 0.39 \\
\hline $\mathrm{G} \times \mathrm{C} \times \mathrm{S}$ & 1 & $4.03^{*}$ & 0.56 & $3.00 \#$ & 0.00 & 0.04 \\
\hline \multicolumn{7}{|l|}{ Task III (rotation) } \\
\hline Group (G) & 1 & 1.70 & 1.72 & 2.50 & 0.43 & $3.86^{\#}$ \\
\hline Session (S) & 1 & $13.03 * * *$ & $8.67^{* *}$ & $13.07^{* * *}$ & 0.96 & 1.57 \\
\hline $\mathrm{G} \times \mathrm{S}$ & 1 & $10.74^{* *}$ & $7.76^{* *}$ & $7.02 * *$ & $5.28^{*}$ & 1.29 \\
\hline
\end{tabular}

Note: $F$-values for reaction time (RT), movement time (MT), movement time pen down (MTdown), movement time pen up (MTup) and video reinspection time (MTvideo).

\# $P<0.1,{ }^{*} P<0.05,{ }^{*} * P<0.01,{ }^{* * *} P<0.001$. 
significantly longer than that in the control group. The difference between the groups was slightly reduced from T0 to T1. There was no significant interaction between group and session. However, when we considered the different components of the movement time in detail (Fig. 4), there was a highly significant decrease in the movement time pen up (MTup) in the patient group.

There were no differences in the number or type of errors between the patient group and the control group at $\mathrm{T} 0$ or $\mathrm{T} 1$.

\subsubsection{Task III: Rotation}

The mean reaction and movement times at $\mathrm{T} 0$ and $\mathrm{T} 1$ are presented in Fig. 2 and the results of the statistical tests in Table 1 . The reaction time in the patient group decreased significantly more than the reaction time in the control group from $\mathrm{T} 0$ to $\mathrm{T} 1$. At $\mathrm{T} 1$, the reaction time in the patient group was not significantly shorter than the reaction time in the control group. The overall (T0 and T1) group difference was not significant, while the interaction between group and session was clearly significant.
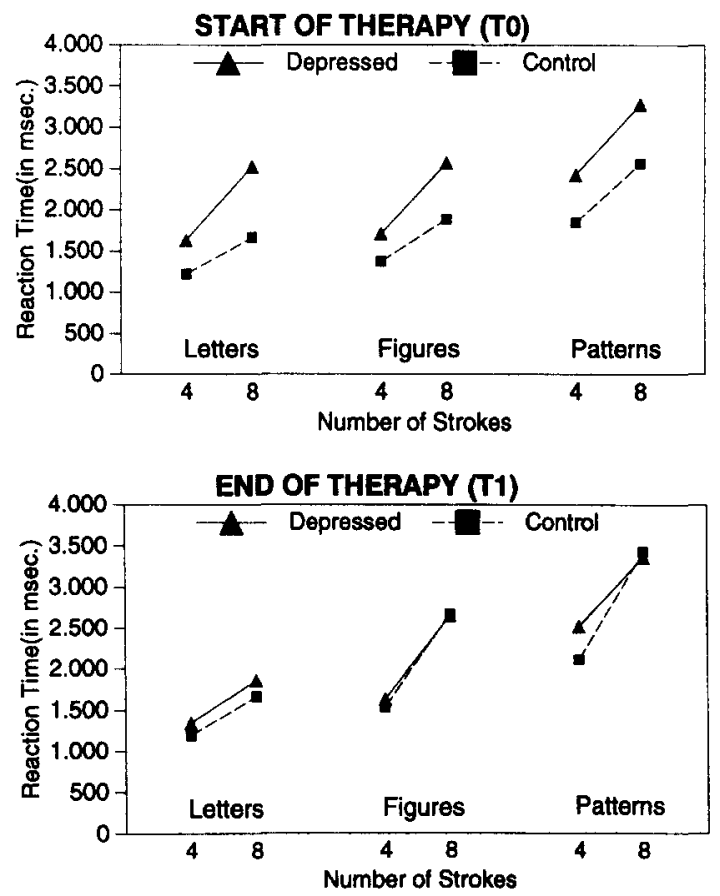

Fig. 3. Mean reaction times (RT) of the depressed patients and the controls at T0 (top panel) and T1 (bottom panel) for copying complex figures in Task II.

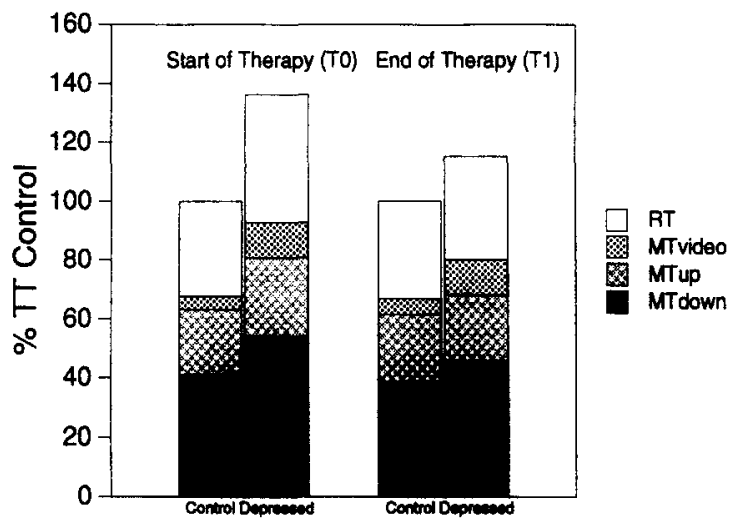

Fig. 4. Mean total time (TT), mean reaction time (RT) and mean movement time (MT), divided into mean video reinspection time (MT video), mean movement time pen up (MTup) and mean movement time pen down (MTdown), of the depressed patients and the controls at $\mathrm{T} 0$ and $\mathrm{T} 1$ for copying complex figures in Task II. Because of a more difficult test set at $\mathrm{T} 1$ than at $\mathrm{T} 0$, all values are presented as percentages of the total time of the control group, scored as $100 \%$ at $\mathrm{T} 0$ and $\mathrm{T} 1$.

The pattern was similar for the movement time: the movement time in the patients decreased from $\mathrm{T} 0$ to $\mathrm{Tl}$, while that of the controls remained unchanged. The overall group difference was not significant. For the total movement time and for the movement time pen up (MTup) the interactions between group and session were highly significant. They also reached significance for the movement time pen down (MTdown).

There were no difference in the number or type of errors between the patient group and the control group at $\mathrm{T} 0$ or $\mathrm{T} 1$.

\section{Discussion}

When we considered the group differences between the patient group and the control group at the start of treatment and at the end of the course 5 weeks later, the results were very univocal for nearly all drawing and copying tasks. There was a decrease in the reaction time in the patient group for copying lines, simple figures, complex figures and for drawing figures after rotation. In task III there was also a significant decrease in the movement time. Patients did not make significantly more or significantly fewer errors than the control subjects either at the start or at the end of treatment. Only differences in speed were found, not in accuracy. 
If we take a decrease in the HDRS score of at least $40 \%$, combined with a final maximum HDRS score of 18 as criteria for successful treatment, eleven patients had a successful outcome, while ten patients had not improved. The reaction times in the success group at $\mathrm{T} 0$ for tasks I (lines-simple figures) and II (complex figures) were significantly or nearly significantly longer than those in the failure group. For the simple figures, the RT in the success group was 1264 $\mathrm{ms}$, while the RT in the failure group was $885 \mathrm{~ms}$. From $\mathrm{T} 0$ to $\mathrm{Tl}$ there was a significant decrease in the reaction time in the success group (from 1264 to $1048 \mathrm{~ms}$ ). This was not the case in the failure group (from 855 to $788 \mathrm{~ms}$ ). The difference in the amount of decrease between the two groups (interaction between group and session) did not reach significance. When we considered the success group in detail, it appeared that five patients showed clear remission. They all had major depression with psychotic features. The other six improvers showed less clear improvement. They all had a major depression, melancholic type. The improvement in the kinematic variables for these subgroups (improved psychotic patients, improved melancholic patients and non-improved patients) corresponded with clinical improvement. The decrease in reaction time and movement time in all tasks was largest in the psychotic depressive subgroup, moderate in the improved melancholic patients and absent in the non-improved patients. The interactions between subgroup and session did not reach significance, with the exception of the MT and the MTup in the copying of the complex figures (task II). These results underline the importance of psychomotor disturbance in psychotic and melancholic depression (Parker et al., 1993, 1994).

In answer to the second question regarding the nature of this reduction in fine motor retardation, the results clearly indicated an improvement in the socalled cognitive processes. This could be concluded from both strategies that were followed: analysis of the kinematic variables and manipulation of the cognitive and motor demands in the different tasks.

First, it was demonstrated in all tasks that the differences in reaction time between the patient group and the control group decreased significantly from T0 to T1 (significant interactions between group and session). The reaction time mainly reflects cognitive processing. In contrast, e.g., in tasks I and II there was no such a decrease in the differences in movement time, which indicates that the motor deficit itself, i.e., slowing of the motor processes, remained unchanged after 6 weeks of treatment. However, when we analysed this movement time in the complex figures in detail, it appeared that the movement time pen up decreased significantly between $\mathrm{T} 0$ and $\mathrm{T} 1$. This component mainly reflects the planning and programming processes, i.e., processes of a cognitive nature.

The other approach to the second question was to manipulate independent variables that are assumed to affect either cognitive or motor processes. In task I, it appeared that the reduction in reaction time between T0 and T1 was greater for the diagonal lines than for the vertical and horizontal lines. On a cognitive level, i.e., in visuo-spatial processing and planning, these diagonal lines are more difficult to draw than the orthogonal lines (Hulstijn et al., 1994). Complexity, which is considered to be a cognitive variable, was manipulated by augmenting the number of strokes in the figures in task I (simple figures) and task II (complex figures). In task II familiarity was changed by introducing figures that were perceptually well-known but motorically not practised, and totally unknown patterns. These stimuli were alternated with letters that were perceptually and motorically well-known. In both tasks, complexity clearly had more affected the reaction time in the patient group than the reaction time in the control group. The patients had greater difficulty with accomplishing the more complex figures than the control group. After treatment, the patients had far less difficulty in task I and no difficulty in task II. In task III, there was a decrease in the reaction time, movement time, and the components the movement time pen up and the movement time pen down during treatment. We considered that this task was fundamentally different from the other tasks: it required a very complex visuo-spatial manoeuvre, that demanded greater cognitive effort. This caused slowing of all the components of the movement; during the movement time pen down, more 'cognitive' readjustements (hand-eye coordination) probably also had to be made. Consequently, the results of this task have to be interpreted differently and they do not allow us to distinguish between the different processes involved. 
It can be concluded from the analysis of the movement itself and manipulation of the different cognitive and motor variables that the reduction in psychomotor slowing after treatment was essentially the result of faster cognitive processing. The initial motor deficit remained unchanged and was responsible for the main difference that existed at the end of treatment between the patients and the controls. The changes in reaction time confirmed earlier results obtained with other methods of measuring psychomotor retardation. For example, elongation of the Speech Pause Time disappeared as the patients' condition improved after treatment with tricyclic antidepressants or electroconvulsive therapy (Greden et al., 1981). At present we have too little understanding of this reduction or disappearance of cognitive slowing and of the different psychological functions involved. It is also unclear whether the subsisting motor deficit is a state or trait marker and whether motor retardation changes during further treatment.

Careful measurement and detailed analysis of fine motor behaviour during drawing and copying tasks will probably provide more precise answers to these questions and enhance the predictive validity of psychomotor variables in the antidepressant treatment response.

\section{Acknowledgements}

We thank Marion Pagen for helping to set up the experiment, Ans Lenders and Jacky Mullekom for testing part of the subjects, and Bea Kosterman, Arjen Lelivelt, Lydia Krabbendam and Annemiek Beemsterboer for their assistance in the statistical analyses. We thank Mrs. E. van Bergen for her secretarial assistance. This study was supported in part by a grant of Eli Lilly Nederland.

\section{References}

American Psychiatric Association (1987) Diagnostic and statistical manual fo mental disorders (3rd Edn.-Revised). American Psychiatric Association, Washington, DC.

Ghozlan, A. and Widlöcher, D. (1989) Decision time and movement time in depression: Differential effects of practice before and after clinical improvement. Percept. Motil. Skills 68, 187-192.
Greden, J.F. and Carroll, B.J. (1980) Decrease in speech pause times with treatment of endogenous depression. Biol. Psychiatry $15,575-587$.

Greden, J.F., Albala, A.A., Smokler, I.A., Gardner, R. and Carroll, B.J. (1981) Speech pause time: a marker of psychomotor retardation among endogenous depressives. Biol. Psychiatry 16, 581-589.

Hamilton, M. (1960) A rating scale for depression. J. Neurol. Neurosurg. Psychiatry 23, 56-62.

Hulstijn, W., Van Mier, H.I.A.J. and Van Hoof, J.J.M. (1994) Figure copying and psychomotor retardation in depression. In C. Faure, P. Keuss, G. Lorette and A. Vinter (Eds.), Advances in handwriting and drawing: A multidisciplinary approach. Europia, Paris, pp. 477-488.

Hoffmann, G.M.A., Gonze, J.C. and Mendlewicz, J. (1985) Speech pause time as a method for the evaluation of psychomotor retardation in depressive illness. Br. J. Psychiatry 146, 535538.

Joyce, P.R. and Paykel, E.S. (1989) Predictors of drug response in depression. Arch. Gen. Psychiatry 46, 89-99.

Kuny, S. and Stassen, H.H. (1993) Speaking behavior and voice sound characteristics in depressive patients during recovery. J. Psychiatr. Res. 27, 289-307.

Maarse, F.J., Janssen, H.J.J. and Dexel, F. (1988) A special pen for an XY-tablet. In F.J. Maarse, J.M. Mulder, W.P.B. Sjouw and A.E. Akkerman (Eds.) Computers in Psychology: Methods, Instrumentation and Psychodiagnostics. Swets and Zeitlinger, Amsterdam, pp. 133-139.

Parker, G., Hadzi-Pavlovic, D., Brodaty, H., Boyce, P., Mitchell, P., Wilhelm, K., Hickie, I. and Eyers, K. (1993) Psychomotor disturbance in depression: defining the constructs. J. Affect. Disord. 27, 255-265.

Parker, G., Hadzi-Pavlovic, D., Wilhelm, K., Hickie, I., Brodaty, H., Boyce, P., Mitchell, P. and Eyers, K. (1994) Defining melancholia: properties of a refined sign-based measure. $\mathrm{Br}$. J. Psychiatry 164, 316-326.

Rogers, D., Lees, A.J., Smith, E., Trimble, M. and Stern, G.M. (1987) Bradhyphrenia in Parkinson's disease and psychomotor retardation in depressive illness. Brain 110, 761-776.

Royant-Parola, A.A., Borbely, I., Tobler, O., Benoit and Widlöcher, D. (1986) Monitoring of long-term motor activity in depressed patients. Br. J. Psychiatry 149, 288-293.

Sabbe, B., Hulstijn, W., Van Hoof, J.J.M and Zitman, F.G. (1996). Fine motor retardation and depression. J. Psychiatr. Res., in press.

Van Hoof, J.J.M., Hulstijn, W., Van Mier, J.I.A.J. and Pagen, M. (1993) Fine drawing and psychomotor retardation: Preliminary results. J. Affect. Disord. 23, 263-266.

Van Mier, J.I.A.J. and Hulstijn, W. (1993) The effects of motor complexity on initiation time in writing and drawing. Acta Psychol, 84, 231-251.

Widlöcher, D. (1983a) Psychomotor retardation: clinical, theoretical and psychometric aspects. Psychiatr. Clin. North Am. 6, $27-40$.

Widlöcher, D. (1983b) Retardation: A basic emotional response? In J.M. Davis and J.W. Maas (Eds.), The affective disorders. American Psychiatric Press, Washington, DC, pp. 165-181.

Zung, W.K. (1965) A self-rating depression scale. Arch. Gen. Psychiatry 12, 63-70. 